\title{
Fractal or biologically variable delivery of cardioplegic solution prevents diastolic dysfunction after cardiopulmonary bypass
}

\author{
M. R. Graham, MDa \\ R. K. Warrian, MD ${ }^{\text {b }}$ \\ L. G. Girling, BSc ${ }^{a}$ \\ L. Doiron, $\mathrm{CPC}^{\mathrm{b}}$ \\ G. R. Lefevre, $M D^{\mathrm{a}}$ \\ M. Cheang, MMath ${ }^{\mathrm{C}}$ \\ W. A. C. Mutch, $M D^{\mathrm{a}}$
}

\footnotetext{
From the Departments of Anesthesia, Cardiovascular Surgery, ${ }^{b}$ and Community Health Sciences, ${ }^{c}$ University of Manitoba, Winnipeg, Manitoba, Canada.

The Crocus Investment Fund and the Industrial Research Assistance Program provided funding. Some of the concepts discussed are protected by US Patents $\# 5,647,350$, \#5,941,841 and \#6,027,498; "Control of Life Support Systems," owned by Biovar Life Support Inc, jointly held by Drs Mutch, Lefevre, the University of Manitoba, and the Crocus Investment Fund.

Received for publication April 23, 2001; revisions requested June 7, 2001; revisions received June 21, 2001; accepted for publication June 26, 2001

Address for reprints: W. A. C. Mutch, MD, Professor, Department of Anesthesia, University of Manitoba, A504 Chown Building, c/o 170 Services Building, 744 Bannatyne Ave, Winnipeg, Manitoba, Canada R3C 0W3 (E-mail: amutch@ms. umanitoba.ca).

J Thorac Cardiovasc Surg 2002;123:63-71

Copyright (C) 2002 by The American Association for Thoracic Surgery

0022-5223/2002 \$35.00 + $0 \quad \mathbf{1 2 / 1 / 1 1 8 2 7 7}$

doi:10.1067/mtc.2002.118277
}

Objective: To determine whether myocardial protection is improved by restoring physiologic variability to the cardioplegia pressure signal during cardiopulmonary bypass, we compared cardiac function in pigs in the first hour after either conventional cold-blood cardioplegia (group CC) or computer-controlled biologically variable pulsatile cardioplegia (group BVC).

Methods: Invasive monitors and sonomicrometry crystals were placed, and cardiopulmonary bypass was initiated. The aorta was crossclamped, and cold blood cardioplegic solution was infused intermittently through the aortic root with either conventional cardioplegia $(n=8)$ or biologically variable pulsatile cardioplegia $(n=8$; mean pressure, $75 \mathrm{~mm} \mathrm{Hg}$ for 85 minutes). The crossclamp was released, cardiac function was restored, and separation from cardiopulmonary bypass was completed. With stable temperature and arterial blood gases, hemodynamics and systolic and diastolic indices were compared at 15, 30, and 60 minutes after cardiopulmonary bypass.

Results: Diastolic stiffness doubled from $0.027 \pm 0.016 \mathrm{~mm} \mathrm{Hg} / \mathrm{mm}$ (mean $\pm \mathrm{SD}$ ) at baseline to $0.055 \pm 0.036 \mathrm{~mm} \mathrm{Hg} / \mathrm{mm}(P=.003)$ at 1 hour after bypass in group $\mathrm{CC}$, associated with increased left ventricular end-diastolic pressure from $9 \pm 2$ to $11 \pm 2 \mathrm{~mm} \mathrm{Hg}(P=.001)$, mean pulmonary artery pressure from $14 \pm 2$ to $20 \pm 3$ $\mathrm{mm} \mathrm{Hg}(P=.003)$, and serum lactate levels from $2.0 \pm 0.5$ to $5.6 \pm 2.3 \mathrm{mmol} / \mathrm{L}(P$ $=.008)$. Systolic function was not affected. In group BVC diastolic stiffness, left ventricular end-diastolic pressure, and pulmonary artery pressure values were not different from control values at any time after bypass, and serum lactate levels were significantly less than with conventional cold blood cardioplegia. Peak pressure variability with biologically variable pulsatile cardioplegia fit a power-law equation (exponent $=-3.0 ; R^{2}=0.97$ ), indicating fractal behavior.

Conclusion: Diastolic cardiac function is better preserved after cardiopulmonary bypass with biologically variable pulsatile cardioplegia and fractal perfusion. This may be attributed to enhanced microcirculatory perfusion with improved myocardial protection. A model supporting these results is presented.

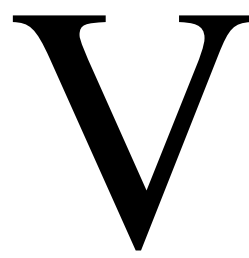

entricular dysfunction after cardiac surgery may be caused by incomplete myocardial protection during cardiopulmonary bypass (CPB) and aortic crossclamping. Optimal myocardial protection depends on adequate delivery of cardioplegia solution to the entire coronary microcirculation, but coronary blood flow distribution is heterogeneous under normal conditions. ${ }^{1-3}$ With max- 
imal coronary vasodilation induced pharmacologically, 4fold differences in blood flow between regions can be demonstrated, and flow variability increases at lower perfusion pressures. ${ }^{4}$

The distribution of cardioplegic solution is also heterogeneous, even in the absence of coronary disease, and Aldea and colleagues ${ }^{5}$ have emphasized the importance of coronary perfusion pressure on delivery of cardioplegic solution. Standard cardioplegia is delivered with a roller pump with an apulsatile flow pattern. This may not optimally recruit all vascular territories during ischemic arrest, with the attendant increase in vascular resistance and vessel closure. Thus, there is a high likelihood that some regions of the myocardium may receive inadequate protection with standard cardioplegia.

Bassingthwaighte and colleagues ${ }^{6,7}$ have suggested that the branching pattern of the coronary circulation is fractal in structure, which may explain the heterogeneous flow patterns observed. Fractals are structures or processes that demonstrate self-similarity with increasing magnification. Global and regional pressure flow from the myocardium can be mathematically modeled as simple power-law functions with differing exponents. ${ }^{5,8,9}$ Such power-law functions also indicate fractal behavior.

We have developed biologically variable (BV) life support, which restores the normal variability seen in physiologic signals to either roller pumps or mechanical ventilators by using computer controllers. ${ }^{10-15} \mathrm{BV}$ life support may be characterized as a fractal process because the pressure-time relationships generated by either of the computer controllers also fit power-law functions.

In the vascular system BV CPB results in improved cerebral oxygenation during rewarming from moderate hypothermia compared with either apulsatile ${ }^{11}$ or monotonously regular pulsatile CPB. ${ }^{14}$ Mathematic modeling suggests that biologic variability, using a fractal sequence for the driving pressure, will augment recruitment of either alveoli in the respiratory system ${ }^{16}$ or microvessels in the vascular system ${ }^{14}$ without an overall increase in driving pressure.

We hypothesized that infusing cardioplegic solution in a fractal manner may enhance delivery, resulting in improved myocardial protection during ischemic arrest.

\section{Materials and Methods}

The experiments were approved by the Committee for Animal Experimentation at the University of Manitoba, and animals were handled according to the standards of the Canadian Council on Animal Care.

\section{Surgical Preparation}

Twenty healthy pigs (33-38 kg) were premedicated (atropine, 0.6 $\mathrm{mg}$; ketamine, $12 \mathrm{mg} / \mathrm{kg}$; and midazolam, $0.5 \mathrm{mg} / \mathrm{kg}$ ) and anesthetized with isoflurane $1.4 \%$ in oxygen, with doxacurium (8 $\mathrm{mg} / \mathrm{h}$ ) used for muscle relaxation. Volume-cycled ventilation through an endotracheal tube maintained arterial carbon dioxide and $\mathrm{pH}$ within the normal range. Electrocardiograms were recorded with surface electrodes.

Femoral arterial, pulmonary arterial, and urinary catheters were placed to monitor mean arterial blood pressure, pulmonary artery pressure, and urine volume. Through the femoral vein, an $8 \mathrm{~F}$ balloon-tipped Fogarty catheter was passed into the inferior vena cava above the level of the diaphragm. Nasopharyngeal and left ventricular myocardial free-wall temperature probes were positioned for continuous temperature monitoring.

A sternotomy was performed, and the pericardium was opened. Left ventricular dimension was measured in 2 planes (anteriorposterior and apex-base) by pairs of 2-mm piezo-electric ultrasonic transducers positioned on the endocardial surface through a stab wound and secured with a purse-string suture. A Millar Mikro-tip 3F catheter (Millar Instruments, Inc, Houston, Tex) was secured in the left ventricle through the apex for continuous measurement of left ventricular pressure (LVP). The crystal pairs were attached to a digital sonomicrometer (Sonometrics Corporation, London, Ontario, Canada).

CPB cannulas were placed in the ascending aorta $(6.5 \mathrm{~mm}$; Stöckert GmbH, Freiburg, Germany) and right atrium (32F DLP single-stage cannula; DLP, Inc, Walker, Mich). A 9F cannula (Medtronic, Inc, Minneapolis, Minn) with a side branch for pressure monitoring was inserted into the aortic root for administration of cardioplegic solution. Before the start of CPB, $100 \mathrm{mg}$ of sodium hydrocortisol succinate was given intravenously. The bypass circuit was primed with lactated Ringer solution $(500 \mathrm{~mL})$, pentastarch (Pentaspan; $500 \mathrm{~mL}$ ), and mannitol (Osmitrol; 500 $\mathrm{mL}$ ) with $5000 \mathrm{IU}$ of heparin to a total volume of $1.5 \mathrm{~L}$. Animals were then randomized to receive either conventional cold-blood cardioplegia $(\mathrm{CC})$ or biologically variable cardioplegia (BVC; water bath temperature $=0^{\circ} \mathrm{C}$ ). All hemodynamic parameters were recorded simultaneously on a 6-channel chart recorder (Gould Instrumentation Systems, Valley View, Ohio) and data-acquisition system (Dataq, Codas). Echocardiography, LVP, and sonomicrometry crystal data were monitored continuously on-line to the Sonometrics computer screen and intermittently recorded for offline analysis.

After heparinization to an activated clotting time of greater than 400 seconds, CPB was initiated with a flow rate of approximately $3 \mathrm{~L} / \mathrm{min}$. We used a Cobe apulsatile roller pump, membrane oxygenator (Cobe Optima Hollow Fiber Open System; Cobe Cardiovascular, Inc, Arvada, Colo), and arterial in-line filter (Baxter 1040D 40- $\mu \mathrm{m}$ pore; Baxter Healthcare Corp, Santa Ana, Calif) with alpha-stat $\mathrm{pH}$ management. Normothermic bypass was maintained for approximately 15 minutes to ensure stable hemodynamics and blood gases before placement of the aortic crossclamp. No vasoactive drugs were used during the bypass run. The animals were then actively cooled to $33^{\circ} \mathrm{C}$.

\section{Protocol}

Control measurements were taken after cannula placement and before initiation of CPB. After aortic crossclamping, cardioplegia was standardized to 20-minute cycles consisting of 5 minutes of either CC or BVC cardioplegia at a mean perfusion pressure of 75 
$\mathrm{mm} \mathrm{Hg}$ and 15 minutes of no perfusion. Five cardioplegia cycles resulted in a total crossclamp time of 85 minutes. We used 1 minute of high-concentration $\mathrm{KCl}$ solution $(80 \mathrm{mEq} / \mathrm{L})$ and $4 \mathrm{~min}$ utes of low-concentration $\mathrm{KCl}$ solution $(30 \mathrm{mEq} / \mathrm{L})$ at a ratio of 4:1 blood/cardioplegic solution as the perfusing solution.

The computer controller and software for biologically variable control of the roller pump have been described previously. ${ }^{11}$ In brief, the systolic variations in arterial blood pressure obtained from a lightly anesthetized, spontaneously breathing pig were used to create the variability file. Minimum, maximum, and mean values for each peak systolic arterial pressure were calculated. The minimum pressure was subtracted from each pressure, and the new minimum $(0 \mathrm{~mm} \mathrm{Hg})$, maximum, and mean peak values were recalculated and displayed. After initiation of cardioplegia, the target mean cardioplegia pressure and maximum amount of computer modulation were set. We chose a mean perfusion pressure of $75 \pm 25 \mathrm{~mm} \mathrm{Hg}$ pulse variation generated by the computer controller. The roller-pump flow rate was adjusted to achieve the minimum pressure of $50 \mathrm{~mm} \mathrm{Hg}$, with the computer modulation level preset to zero. The modulation level was then adjusted until the increase in pump flow rate resulted in an increase in cardioplegia pressure from 50 to $100 \mathrm{~mm} \mathrm{Hg}$, the maximum computer modulation level chosen. Computer control of the roller pump was now initiated. At any time, independent setting of the rate control could adjust mean cardioplegia pressure to the predetermined level if required. This setup was complete within 1 minute of initiation of cardioplegia in all cases. Figure 1 shows the pressure profile obtained in the aortic root with either CC or BVC over 35 seconds.

Lidocaine ( $2 \mathrm{mg} / \mathrm{kg}$ administered intravenously) was given 2 minutes before crossclamp release. At the end of the fifth cardioplegia administration, the crossclamp was released, and cardiac activity was restored spontaneously or with internal defibrillation (10-30 J) if necessary. No inotropic or vasoactive drugs were used. A volume-recruitment maneuver of 3 vital capacity breaths was performed, mechanical ventilation was resumed, and separation from bypass was achieved.

Arterial blood gases, hemodynamics, temperatures. and cardiac function were determined at 15, 30, and 60 minutes after $\mathrm{CPB}$. The animals were then killed with an injection of sodium pentobarbital (Euthanol; $100 \mathrm{mg} / \mathrm{kg}$ ).

\section{Arterial Blood Gas, Temperature, and Hemodynamic Measurements}

At each measurement period, arterial and mixed venous blood gases were sampled, and temperature was recorded. Heart rate, mean arterial pressure, mean pulmonary artery pressure, left ventricular end-diastolic pressure (LVEDP), and left ventricular enddiastolic volume (LVEDV) were measured, and cardiac output was determined.

\section{Cardiac Function Determinations}

At each measurement period, LVEDV was standardized visually to control levels with transfusion. A recording of 15 to 20 heart beats was made at end-expiration. The balloon tip of the inferior vena cava catheter was then inflated for approximately 30 seconds to transiently decrease left ventricular filling so that a range of LVPs at decreasing left ventricular dimensions could be obtained.

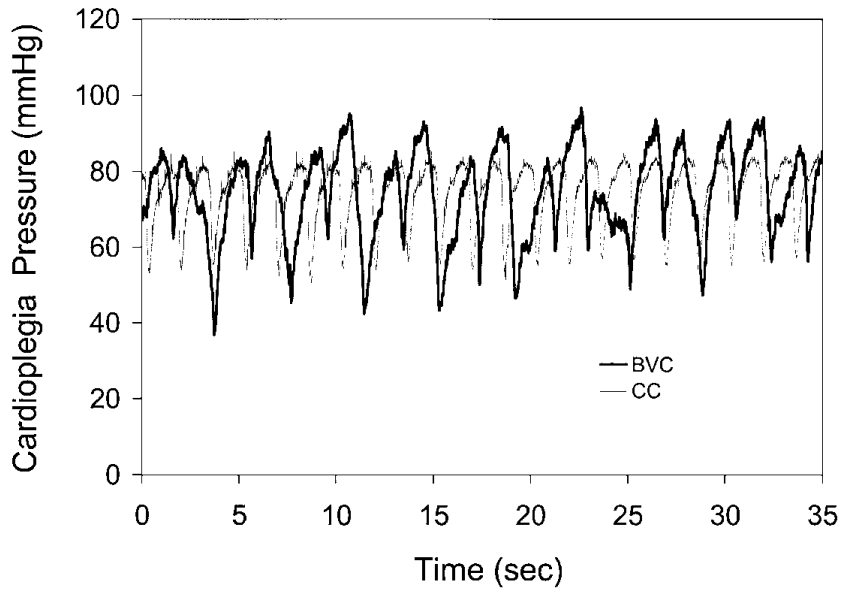

Figure 1. Cardioplegia pressure waveforms as recorded from the cardioplegia cannula to the data-acquisition system. Data have been normalized to the same mean pressure for BVC and CC to compare pulse pressures between groups. Data from the BVC experiment are shown as thick black lines, and data from the CC experiment are shown as thin black lines. Sampling rate was every $4 \mathrm{~ms}$ for each curve.

\section{Analysis}

Postexperiment analysis of the cardiac function curves was done with a Sonometrics software package. For each tracing, left ventricular volume (LVV) was calculated from the crystal pairs by using a 2-dimensional ellipsoid model and plotted against LVP.

\section{Systolic Indices}

First, the slope of the end-systolic pressure-volume relationship (Emax) was determined from the cardiac function curves obtained over successive beats of decreasing LVV during inferior vena caval occlusion. End-systole was defined as the point in the cardiac cycle at which elastance (LVP/LVV) was maximal. Emax was determined by means of linear regression.

Second, preload recruitable stroke work index was determined over the same cardiac cycles of decreasing LVV. Stroke work was calculated as the integral of each pressure-volume loop and plotted against the corresponding end-diastolic volume, the slope of which represents preload recruitable stroke work index.

\section{Diastolic Indices}

First, the time constant for ventricular relaxation $(\tau)$ was calculated from the decline in the LVP pressure trace at stable LVEDP and end-expiration by the following equation:

$$
\mathrm{P}_{\mathrm{t}}=\mathrm{P}_{0} \mathrm{e}^{(-\mathrm{t} / \tau)}
$$

where $\mathrm{P}_{\mathrm{t}}$ is LVP at any time $\mathrm{t}$, and $\mathrm{P}_{0}$ is LVP at time $0 . \mathrm{P}_{0}$ was determined at minimal $\mathrm{dP} / \mathrm{dt}$, the start of isovolumic relaxation. The relationship was determined with a floating asymptote, and $\tau$ was calculated as the negative reciprocal of the slope of the following relationship:

$$
\ln \mathrm{P}_{\mathrm{t}}=-\mathrm{t} / \tau+\ln \mathrm{P}_{0}
$$


TABLE 1. Temperatures and blood gases

\begin{tabular}{|c|c|c|c|c|}
\hline & Before CPB & 15 min after CPB & 30 min after CPB & 60 min after CPB \\
\hline \multicolumn{5}{|l|}{$\mathrm{T}_{\mathrm{n}}\left({ }^{\circ} \mathrm{C}\right)$} \\
\hline CC & $36.1 \pm 0.2$ & $35.8 \pm 0.4^{*}$ & $35.7 \pm 0.5^{*}$ & $35.5 \pm 0.6^{*}$ \\
\hline BVC & $35.9 \pm 0.3$ & $35.6 \pm 0.3^{*}$ & $35.4 \pm 0.4^{*}$ & $35.5 \pm 0.5^{*}$ \\
\hline \multicolumn{5}{|l|}{$\mathrm{T}_{\mathrm{v}}\left({ }^{\circ} \mathrm{C}\right)$} \\
\hline $\mathrm{CC}$ & $36.2 \pm 0.3$ & $35.9 \pm 0.4$ & $35.8 \pm 0.4$ & $36.0 \pm 0.9$ \\
\hline BVC & $36.1 \pm 0.3$ & $35.6 \pm 0.3$ & $35.5 \pm 0.4^{*}$ & $35.6 \pm 0.5^{*}$ \\
\hline \multicolumn{5}{|c|}{$\mathrm{PaO}_{2}(\mathrm{~mm} \mathrm{Hg})$} \\
\hline CC & $500 \pm 46$ & $415 \pm 129$ & $428 \pm 107$ & $451 \pm 68$ \\
\hline BVC & $520 \pm 31$ & $415 \pm 67$ & $454 \pm 53$ & $475 \pm 32$ \\
\hline \multicolumn{5}{|c|}{$\mathrm{PaCO}_{2}(\mathrm{~mm} \mathrm{Hg})$} \\
\hline $\mathrm{CC}$ & $39 \pm 3$ & $41 \pm 6$ & $38 \pm 8$ & $39 \pm 4$ \\
\hline BVC & $39 \pm 3$ & $40 \pm 5$ & $41 \pm 3$ & $39 \pm 2$ \\
\hline \multicolumn{5}{|c|}{ Arterial pH } \\
\hline $\mathrm{CC}$ & $7.43 \pm 0.04$ & $7.30 \pm 0.07^{*}$ & $7.33 \pm 0.08^{*}$ & $7.32 \pm 0.04^{*} \dagger$ \\
\hline BVC & $7.43 \pm 0.05$ & $7.34 \pm 0.07^{*}$ & $7.34 \pm 0.05^{*}$ & $7.37 \pm 0.05^{*}$ \\
\hline
\end{tabular}

There were 8 animals in each group. Values are given as means \pm SD. CPB, Cardiopulmonary bypass; $T_{n^{\prime}}$ nasopharyngeal temperature; $T_{v}$, central venous temperature; $\mathrm{PaO}_{2^{\prime}}$ arterial partial pressure of oxygen; $\mathrm{PaCO}_{2^{\prime}}$ arterial partial pressure of carbon dioxide.

${ }^{*} P<.05$ within groups versus before CPB.

$\dagger P<.05$ between groups.

TABLE 2. Hemodynamics

\begin{tabular}{|c|c|c|c|c|}
\hline & Before CPB & $15 \mathrm{~min}$ after CPB & $30 \mathrm{~min}$ after CPB & $60 \mathrm{~min}$ after CPB \\
\hline \multicolumn{5}{|c|}{ Heart rate (beats/min) } \\
\hline CC & $100 \pm 17$ & $123 \pm 35^{*}$ & $120 \pm 29^{*}$ & $118 \pm 22^{*}$ \\
\hline BVC & $102 \pm 14$ & $116 \pm 14^{*}$ & $116 \pm 15^{*}$ & $116 \pm 14^{*}$ \\
\hline \multicolumn{5}{|c|}{$\operatorname{MAP}(\mathrm{mm} \mathrm{Hg})$} \\
\hline CC & $70 \pm 8$ & $62 \pm 11^{*}$ & $64 \pm 15$ & $63 \pm 14$ \\
\hline BVC & $68 \pm 8$ & $62 \pm 10$ & $61 \pm 8^{*}$ & $61 \pm 8^{*}$ \\
\hline \multicolumn{5}{|c|}{ Cardiac output (L/min) } \\
\hline $\mathrm{CC}$ & $3.2 \pm 0.9$ & $3.2 \pm 0.9$ & $3.4 \pm 1.0$ & $3.7 \pm 1.2$ \\
\hline BVC & $3.0 \pm 0.7$ & $3.7 \pm 1.1$ & $3.5 \pm 1.1$ & $3.4 \pm 0.7$ \\
\hline \multicolumn{5}{|c|}{ PAP $(\mathrm{mm} \mathrm{Hg})$} \\
\hline CC & $14 \pm 2$ & $19 \pm 3^{*} \dagger$ & $19 \pm 3^{*} \dagger$ & $20 \pm 3^{*} \dagger$ \\
\hline BVC & $17 \pm 5$ & $16 \pm 4$ & $16 \pm 4$ & $17 \pm 3$ \\
\hline \multicolumn{5}{|c|}{$\operatorname{LVEDP}(\mathrm{mm} \mathrm{Hg})$} \\
\hline CC & $9.9 \pm 2.2$ & $11.3 \pm 2.0^{*} \dagger$ & $11.4 \pm 1.5^{*} \dagger$ & $10.6 \pm 1.7^{*} \dagger$ \\
\hline BVC & $9.8 \pm 1.3$ & $9.2 \pm 1.8$ & $8.8 \pm 0.8$ & $9.3 \pm 1.7$ \\
\hline \multicolumn{5}{|c|}{ LVEDV (mL) } \\
\hline CC & $53.5 \pm 16.8$ & $50.1 \pm 17.1$ & $49.8 \pm 17.1$ & $50.9 \pm 18.4$ \\
\hline BVC & $57.7 \pm 11.8$ & $57.3 \pm 7.5$ & $58.0 \pm 8.7$ & $58.1 \pm 6.8$ \\
\hline
\end{tabular}

There were 8 animals in each group. Values are given as means \pm SD. MAP, Mean arterial pressure; $P A P$, pulmonary artery pressure; $L V E D P$, left ventricular end-diastolic pressure; LVEDV, left ventricular end-diastolic volume.

${ }^{*} P<.05$ within groups versus before $\mathrm{CPB}$.

$\dagger P<.05$ between groups.

Second, LV diastolic stiffness was quantitated by the stiffness constant $(\mathrm{Kp})$ according to the following equation:

$$
\mathrm{LVEDP}=\mathrm{Ce}^{(\mathrm{Kp} \times \mathrm{LVV})}
$$

where $\mathrm{C}$ is a derived constant, $\mathrm{Kp}$ is chamber stiffness, and LVV is the volume corresponding to a given end-diastolic pressure.

\section{Fractal Model of Cardioplegia Administration}

The pressure profile of cardioplegia administration (measured at the cannula site) was analyzed post hoc in both groups. Mean peak systolic arterial pressure (SAP) was obtained in each experiment. Instantaneous pressure measurements (4-ms intervals) were then determined from a representative experiment in both groups. Data 
were compared in the following manner: instantaneous pressure was subtracted from mean peak SAP, and this value was squared and then $\log$ transformed. These data were partitioned into incremental bins of equal size to determine their frequency distribution. The probability of each frequency was determined as $\mathrm{Ni} / \mathrm{N}$, where $\mathrm{Ni}$ is number of observations in a given frequency bin, and $\mathrm{N}$ is total number of observations. A log transformation of the probability distribution was derived. The log probability distribution versus log SAP variation was plotted for the experiment in each group. The equation and correlation coefficient of the data were derived by means of regression analysis.

\section{Cardioplegia Pressure-Flow Model}

The relationship between instantaneous pressure and flow was modeled on the canine results of Aldea and colleagues. ${ }^{5}$ Regional myocardial pressure-flow relationships fit a family of power functions of the form $\mathrm{Q}=\mathrm{P}^{\mathrm{x}}$, with $\mathrm{x}$ varying between 2.07 to 3.42 . We calculated mean cardioplegia flow by averaging instantaneous flow from instantaneous pressures, using these 2 extremes of perfusion. Thirty-five seconds of data from one experiment in each group yielded 8750 instantaneous pressure measurements for this calculation. The $\mathrm{BVC} / \mathrm{CC}$ mean flow ratio was compared at a pressure normalized to 1.0 for each regional flow. Flow was then calculated at $20 \%, 40 \%$, and $50 \%$ of the normalized pressure to mimic the decrease in perfusion pressure that may occur with increasing downstream closing pressure during cardioplegic arrest.

\section{Comparison of Coronary Sinus With Arterial Lactate Concentration}

We conducted 4 additional experiments in an identical model to compare coronary sinus with arterial lactate concentration before and after CPB. A catheter was inserted through the right atrium deep into the coronary sinus by means of direct palpation and confirmed at autopsy. Cardioplegic solution was administered as in group CC. Simultaneous coronary sinus and arterial blood samples were obtained at baseline and after CPB. Lactate concentrations at the 2 sites were compared with linear regression.

\section{Statistics}

Hemodynamic and systolic and diastolic cardiac indices were compared at baseline and at each time period after CPB by a splitplot 1-way analysis of variance for repeated measures. When significant group or group-by-time interactions were found, least squares means test matrices were generated, and within- and between-group comparisons were made. The Bonferroni correction for repeated measures was applied to within-group comparisons. Data are presented as means \pm SD unless otherwise noted.

\section{Results}

Of the 20 pigs studied, 4 were discarded from analysis: 1 before randomization with a severe inflammatory reaction and pulmonary hypertension, $1 \mathrm{CC}$ animal with lobar pneumonia, and 2 BVC animals, 1 for technical concerns and 1 because of aortic incompetence resulting in marked ventricular distention and pulmonary hypertension during cardioplegia. This left 16 animals, 8 in each group, with a complete protocol. Weights were equivalent between the 2

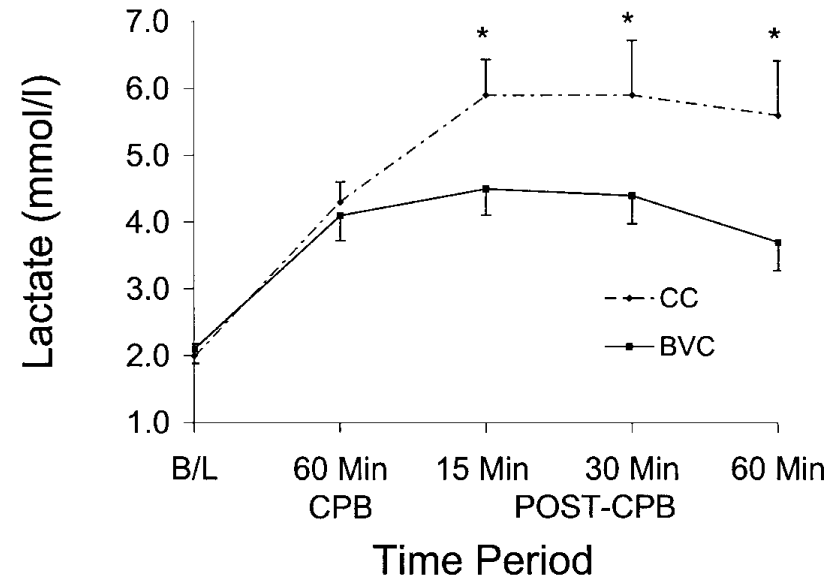

Figure 2. Serum lactate levels over the course of the experiment in both groups at baseline $(B / L$; after cannulation but before $C P B)$, at 60 minutes of $C P B$, and at 15,30 , and 60 minutes after separation from CPB. Lactate levels were significantly greater in group CC compared with those in group BVC at every time period after CPB $(P=.008$, group-by-time interaction, analysis of variance).

groups $(33 \pm 3 \mathrm{~kg}$ and $35 \pm 2 \mathrm{~kg}$ in groups $\mathrm{CC}$ and $\mathrm{BVC}$, respectively; $P=.29$ ).

\section{Temperature, Arterial Blood Gases, and Serum Lactate} Levels

Nasal and venous temperatures and end-tidal isoflurane concentrations were stable, and arterial blood gases were within the normal range at all measurement periods (Table 1). Temperature decreased equally during $\mathrm{CPB}\left(32.9^{\circ} \mathrm{C}\right.$ for the $\mathrm{BVC}$ group and $33.2^{\circ} \mathrm{C}$ for the $\mathrm{CC}$ group), as per the protocol, and was restored to slightly hypothermic levels in both groups after bypass $\left(35.5^{\circ} \mathrm{C}-36^{\circ} \mathrm{C}\right)$. Intramyocardial temperature fell to approximately $8.5^{\circ} \mathrm{C}$ during cardioplegia and rewarmed to approximately $25^{\circ} \mathrm{C}$ between runs, and this was not different between groups. Similar serum lactate levels were present at baseline and after 60 minutes of CPB. After CPB, serum lactate levels were higher in group $\mathrm{CC}$ at every measurement period (group-by-time interaction, $P=$ .008; Figure 2). Comparison of 30 measurements of coronary sinus to arterial lactate concentrations in the 4 post hoc experiments described showed a highly significant correlation $\left(R^{2}=0.97\right)$.

\section{Hemodynamics}

Mean arterial pressure and cardiac output were stable and well maintained at all time periods, with no significant groupby-time interactions (Table 2). A small increase in heart rate was evident in both groups in the postbypass period, with no difference between groups. Pulmonary artery pressure was consistently higher in group CC compared with group BVC at every measurement period after $\mathrm{CPB}$, despite a lower value at baseline (group-by-time interaction, $P=.0003$ ). LVEDP 
TABLE 3. Cardiac indices

\begin{tabular}{lcccc}
\hline & Before CPB & 15 min after CPB & 30 min after CPB & 60 min after CPB \\
\hline Emax $(\mathrm{mm} \mathrm{Hg} / \mathrm{mL})$ & & & & \\
$\quad$ CC & $6.1 \pm 3.2$ & $7.8 \pm 6.2$ & $6.8 \pm 5.9$ & $7.4 \pm 6.4$ \\
$\quad$ BVC & $4.9 \pm 2.6$ & $3.9 \pm 1.8^{*}$ & $4.3 \pm 2.2^{*}$ & $4.7 \pm 2.3^{*}$ \\
PRSWI $(\mathrm{mm} \mathrm{Hg} / \mathrm{mL})$ & & & & \\
$\quad$ CC & $47.7 \pm 8.5$ & $44.3 \pm 11.2$ & $46.1 \pm 11.9$ & $44.5 \pm 9.2$ \\
$\quad$ BVC & $50.4 \pm 6.6$ & $51.0 \pm 6.6$ & $48.0 \pm 9.3$ & $48.2 \pm 10.0$ \\
$\tau \quad$ CC & $37 \pm 7$ & $41 \pm 6$ & & $47 \pm 11$ \\
$\quad$ BVC & $42 \pm 8$ & $44 \pm 14$ & $47 \pm 12$ & $41 \pm 15$ \\
\end{tabular}

There were 8 animals in each group. Values are given as means \pm SD. CPB, Cardiopulmonary bypass; Emax, the slope of the end-systolic pressurevolume relationship; $\tau$, time constant for ventricular relaxation.

${ }^{*} P<.05$ between groups.

was similar between groups at baseline, elevated compared with baseline values at every postbypass period in group CC, and not different from baseline values in group BVC. The group-by-time interaction was not significant, but least squares means analysis indicated a significant difference between groups $\mathrm{CC}$ and $\mathrm{BVC}$ at every measurement period after bypass. LVEDV was well controlled over time in each group, with no group-by-time interaction and no difference between groups. The absolute value of LVEDV was marginally lower in group CC compared with that in group BVC after bypass, whereas the corresponding LVEDP increased; this is evidence of increased ventricular stiffness.

\section{Cardioplegia Parameters}

Mean cardioplegia perfusion pressures were well controlled at $75 \mathrm{~mm} \mathrm{Hg}$ in both groups. Figure 1 represents only 35 seconds of the total data set of 38 minutes for BVC. The SD was 11.7 in group BVC and 8.4 in group $\mathrm{CC}$, resulting in an $\mathrm{SD}$ ratio of 1.4 in this short sequence. The mean pulse pressure was $26 \pm 3 \mathrm{~mm} \mathrm{Hg}$ in group CC as a result of the roller pump alone. For the entire data set, the imposition of the BVC signal resulted in a 2.5 -fold increase of this pulse pressure to $62 \pm 10 \mathrm{~mm} \mathrm{Hg}$.

Volume of cardioplegic solution was not different between groups. Total volumes of high $\mathrm{K}^{+}$solution delivered were $252 \pm 32 \mathrm{~mL}$ in group CC versus $248 \pm 14 \mathrm{~mL}$ in group BVC. Total volumes of low $\mathrm{K}^{+}$solution were $850 \pm$ $134 \mathrm{~mL}$ in group CC versus $922 \pm 98 \mathrm{~mL}$ in group BVC.

\section{Cardiac Events}

Five animals experienced ventricular fibrillation on crossclamp release in each group. Electrical defibrillation was required 12 times in group CC and 16 times in group BVC (not significantly different by the Fisher exact test).

\section{Systolic Function}

Emax was not different at baseline in group CC compared with that in group BVC. The group-by-time interaction was not significant $(P=.26)$, although the absolute value was slightly higher in group $\mathrm{CC}$ at all measurement periods after bypass (Table 3 ). Within groups, there was no significant difference in Emax at any time period compared with baseline values. Preload recruitable stroke work index was equivalent in both groups at baseline and remained unchanged at every measurement period, with no significant group-by-time interactions.

\section{Diastolic Function}

The time constant for ventricular relaxation was not affected by mode of cardioplegia delivery, and no group-bytime interactions were present (Table 3). Kp showed marked differences; it was unchanged at any time period in group BVC but doubled in group CC $(P=.003)$ in the immediate postbypass period and remained significantly elevated at every measurement period after bypass between groups (Figure 3). A significant correlation between the change in diastolic stiffness versus the change in serum lactate level after bypass is present in group CC but absent in group BVC (Figure 4).

\section{Fractal Model of Cardioplegia Administration}

The comparison of fractal behavior of the 2 modes of cardioplegia delivery is shown in Figure 5. With BVC, the power-law exponent was $-3.0\left(R^{2}=0.97\right)$. When data from the experiment in group $\mathrm{CC}$ were examined at the same bin size as in group BVC $\left(100 \mathrm{~mm} \mathrm{Hg}^{2}\right)$, all variation fell within the first bin $(P=1.0)$. In fact, all observations are within $7.6 \mathrm{~mm} \mathrm{Hg}$ of the mean value.

\section{Cardioplegia Pressure-Flow Model}

The ratio of calculated normalized flow between groups $\mathrm{BVC}$ and $\mathrm{CC}$ for each fractional normalized pressure is shown for exponents 3.42 and 2.07 (Figure 6). Curve fitting to the equation $\mathrm{Q}=\mathrm{P}^{3.42}$ demonstrated a mean normalized flow at a normalized pressure of 1.0 to be 1.09 for $\mathrm{BVC}$ and 1.04 for $\mathrm{CC}$. The $\mathrm{BVC} / \mathrm{CC}$ ratio was thus 1.05 , or $5 \%$ greater 


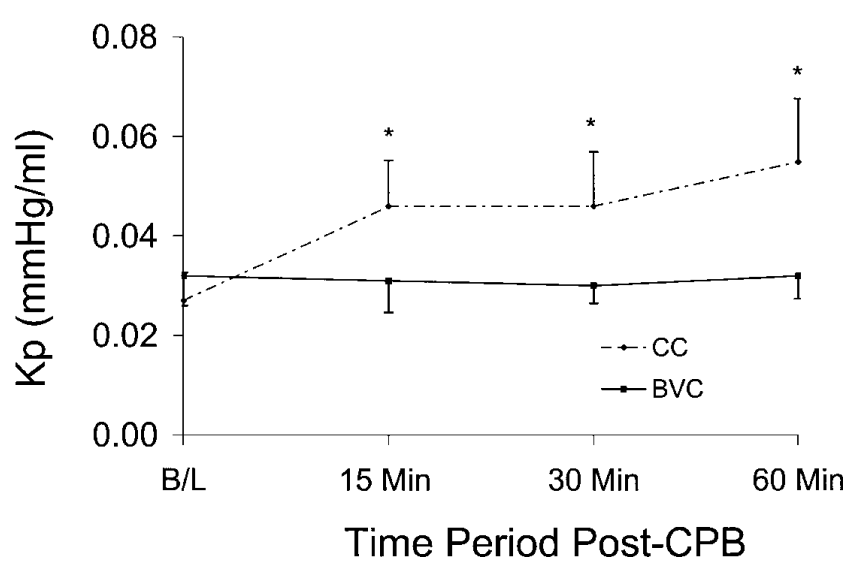

Figure 3. Kp versus time period after CPB. Kp was significantly elevated after bypass in group CC (ie, approximately $100 \%$ increase in stiffness). In contrast, Kp remained essentially unchanged with BVC ( $P=.003$, group-by-time interaction).

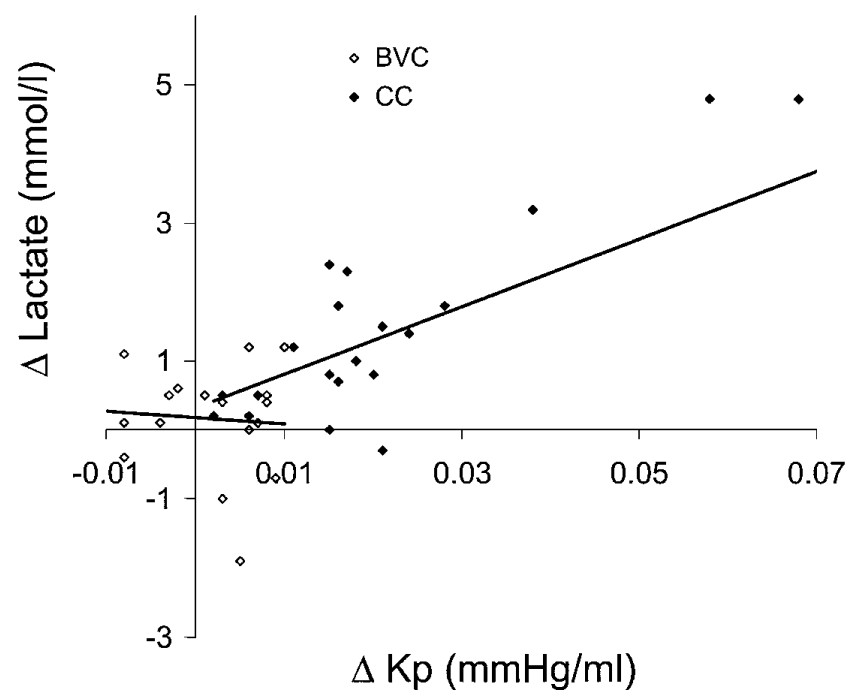

Figure 4. Change in arterial lactate concentration from baseline versus change in Kp from baseline for the 2 experimental groups after separation from CPB. A positive correlation is seen between group CC data between the variables $\left(R^{2}=0.51, P<.01\right)$. No correlation is evident in group BVC $\left(R^{2}=0.01, P=\right.$ not significant).

flow with BVC. With the lower power-law relationship (ie, $\left.\mathrm{Q}=\mathrm{P}^{2.07}\right), 1 \%$ greater flow with $\mathrm{BVC}$ was calculated.

With decreasing normalized perfusion pressures, the $\mathrm{BVC} / \mathrm{CC}$ flow ratio increased. For the higher power-law exponent, at a normalized perfusion pressure of $50 \%$ of baseline, the ratio of calculated normalized flow was $16 \%$ greater with BVC. A more modest effect (5\% greater flow with BVC) was seen with the lower powerlaw exponent.

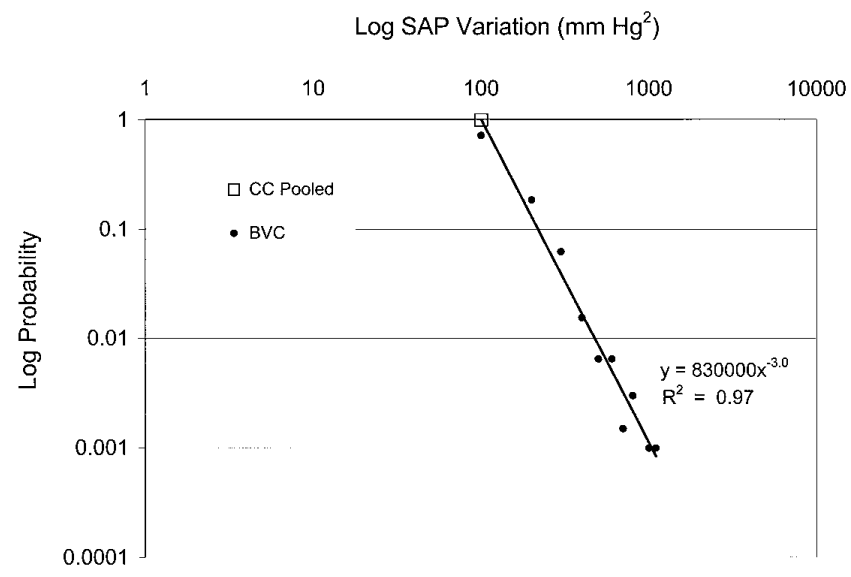

Figure 5. Fractal model of cardioplegia administration with BVC. Analysis of the peak pressure, as measured at the cardioplegia cannula site, is shown for 1 experiment in each group. With BVC, a power-law frequency distribution for log probability of SAP variation versus log SAP variation is seen with a slope of $\mathbf{- 3 . 0}$. Power-law curve fit was an $R^{2}$ value of 0.97 . When examined at the same bin size $\left(100 \mathrm{~mm} \mathrm{Hg}^{2}\right)$, there is no evidence of fractal behavior with CC. This is shown as CC pooled.

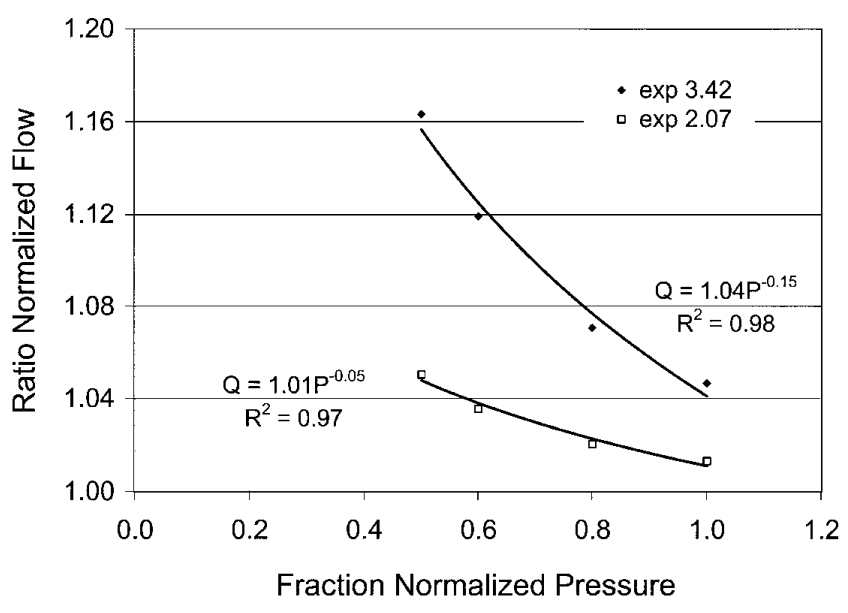

Figure 6. Consequences of fractal or biologically variable delivery of cardioplegic solution with decreasing perfusion pressure. The BVC/CC flow ratio was calculated at $1.0,0.8,0.6$, and 0.5 fractions of normalized pressure for each of 2 power-law curves (ie, exponent 3.42 and 2.07) on the basis of data from Aldea and colleagues. ${ }^{5}$ For both exponents, the normalized BVC/CC flow ratio increases with decreasing fraction of normalized pressure; a greater effect is seen with the higher exponent. Thus, as perfusion pressure decreases, flow is better maintained with BVC. This is most evident in regions of high baseline flow.

\section{Discussion}

This study demonstrates that the pressure profile used to deliver antegrade cardioplegia can influence cardiac function in the first hour after 85 minutes of aortic crossclamp- 
ing and CPB in this porcine model. Systolic function was not affected, but conventional apulsatile cardioplegia (group CC) was associated with increases in diastolic stiffness, LVEDP, pulmonary artery pressure, and serum lactate levels. In contrast, biologically variable cardioplegia delivered in a fractal sequence (group BVC) prevented these changes.

Diastolic dysfunction is an early indicator of myocardial injury and may occur before changes in contractility. Impaired diastolic dysfunction correlates to difficulty in weaning from $\mathrm{CPB}$ and the need for more inotropic support perioperatively. ${ }^{17}$ Increases in diastolic stiffness with standard cardioplegia have been well documented previously, ${ }^{18-20}$ supporting our findings. All 8 animals in group CC demonstrated a sustained increase in Kp after CPB. With LVEDV controlled between measurement periods, LVEDP increased from 9 to $11 \mathrm{~mm} \mathrm{Hg}$ after bypass in group CC, which is further corroboration of a noncompliant ventricle. Neither LVEDV nor pressure were different after bypass in group BVC.

Buckberg $^{21}$ has integrated multiple cardioprotective strategies, including periods of warm- and cold-blood cardioplegia, antegrade and retrograde delivery, and intermittent and continuous perfusion with both blood and crystalloid solutions to improve myocardial protection. A strategy to maximize distribution of cardioplegic solution on the basis of pump-pressure profiles was not considered. We chose a single approach to cardioplegia administration as a model to determine whether the pressure profile for delivery of cardioplegic solution was an important independent parameter. The results support this contention.

Possible mechanisms to explain the benefit of BVC in this setting include better matching of fractal delivery to a fractal vascular bed, facilitation of vascular recruitment, prevention of microvascular closure, and improved flow generated with a noisy pressure profile.

\section{Fractal Model of Cardioplegia Delivery}

Fractal delivery of cardioplegia results in a more variable perfusion pressure, although mean perfusion pressure is identical. Figure 5 shows this difference. When analyzed with the same yardstick of variation (ie, bin sizes of $100 \mathrm{~mm}$ $\mathrm{Hg}^{2}$ ), BVC demonstrated a much noisier signal than that seen with CC. Previous work suggests that collapsed vessels may be better recruited with a noisier input signal. ${ }^{14}$ Suki and colleagues ${ }^{16}$ have suggested that biologically variable life support is an example of stochastic resonance, enhanced output through use of a noisy input signal. Cardioplegia delivery demonstrates nonlinear pressure-flow relationships. ${ }^{5}$ Under such circumstances, a noisier pressure signal results in greater flow because the decrease in flow seen at lower pressures is more than compensated by the increase in flow seen at greater driving pressures. The modeling based on the curves by Aldea and colleagues ${ }^{5}$ bears this out with greater calculated flow at the same mean pressure in group BVC. Importantly, a greater advantage is demonstrated as critical closing pressure increases (Figure 6).

\section{BVC and Critical Closure}

Chen and colleagues ${ }^{22}$ have demonstrated endothelial swelling, resulting in luminal narrowing in myocardial capillaries after cardioplegic arrest. Such changes increase the propensity for vessel closure. Once closed, large increases in pressure are necessary to reinstitute flow. Failure to recruit vascular territories with each administration of cardioplegia would worsen the ischemic insult, creating a positive-feedback loop for myocardial injury. BVC may help prevent this positive-feedback cycle. From the model, as critical closing pressure increases (perfusion pressure 50\% of baseline value), the BVC/CC flow ratio increases (Figure 6). Thus BVC may yield a higher flow at a given perfusion pressure to offer increased myocardial protection. Coggins and colleagues ${ }^{4}$ have shown that at $70 \mathrm{~mm} \mathrm{Hg}, 100 \%$ of left ventricular areas have significant flow reserve, whereas at $30 \mathrm{~mm} \mathrm{Hg}$, equivalent to an increase in critical closing pressure of $40 \mathrm{~mm} \mathrm{Hg}$, only $8 \%$ of regions had flow reserve. Ghaleh and coworkers ${ }^{23}$ showed that areas of high baseline flow, correlating to regions with the highest power-law exponents, are at a 2.3-fold increased risk of myocardial necrosis after 90 minutes of coronary occlusion in the baboon. Thus those regions at greatest risk of ischemia are the same regions that the flow model in Figure 6 would predict are best protected during BVC.

\section{Limitations of the Present Study}

The present study was not designed to measure either microvascular perfusion directly or metabolic markers of coronary perfusion, such as coronary sinus lactate levels. However, evidence of improved coronary perfusion with $\mathrm{BVC}$ is suggested indirectly by the diastolic function and arterial lactate results obtained. The increase in serum lactate levels in group CC cannot be explained by a systematic bias in whole-body perfusion. Hemodynamics were well matched between groups at baseline and during $\mathrm{CPB}$, with similar CPB flow rates and serum lactate levels at 60 minutes of CPB. There was no evidence of impaired systemic perfusion in the postbypass period because heart rate, mean arterial pressure, cardiac index, arterial $\mathrm{PO}_{2}$, mixed venous $\mathrm{PO}_{2}$, and urine output were not different between groups. With no evidence for impaired peripheral perfusion and a strong correlation between the change in diastolic stiffness and serum lactates, as seen in Figure 4, the results suggest that cardiac metabolism contributed to the difference in lactate concentrations seen. Follow-up work indicated a high correlation between arterial and coronary sinus lactate levels in this experimental model, suggesting that the above conclusions are valid. 
We believe that the present study is the first to demonstrate a benefit of increased pulse variability during cardioplegia delivery. Others have shown improved myocardial function with whole-body pulsatile perfusion after various periods of global myocardial ischemia. ${ }^{24-28}$ Although these findings support our conjecture, others have shown no protective effect with whole-body pulsatile perfusion. ${ }^{27}$ The present study in pigs is not directly analogous to the clinical situation. The subjects were healthy young pigs, with no evidence of coronary artery disease or left ventricular dysfunction. As such, we anticipated effects to be small. This was borne out by a reproducible effect on diastolic stiffness alone with a crossclamp time of 85 minutes and intermittent cardioplegia, allowing ventricular activity to recur between cardioplegia runs. However, the majority of patients undergoing bypass have myocardia at greater risk for incomplete or inhomogeneous protection during aortic crossclamping and cardioplegic arrest because of critical coronary stenoses, ventricular dysfunction, and hypertrophy. These preliminary results suggest that administering cardioplegia in a fractal manner by using biologically variable pulsation may enhance myocardial protection.

\section{References}

1. Domenech R, Hoffman J, Noble M, Saunders K, Henson J, Subijanto $\mathrm{S}$. Total and regional coronary blood flow measured by radioactive microspheres in conscious and anesthetized dogs. Circ Res. 1969;25: 581-96.

2. King R, Bassingthwaighte J. Temporal fluctuations in regional myocardial flows. Pflugers Arch. 1989;413:336-42.

3. King R, Bassingthwaighte J, Hales J, Rowell L. Stability of heterogeneity of myocardial blood flow in normal awake baboons. Circ Res. 1985;57:285-95.

4. Coggins DL, Flynn AE, Austin REJ, Aldea GS, Muehrcke D, Goto M, et al. Nonuniform loss of regional flow reserve during myocardial ischemia in dogs. Circ Res. 1990;67:253-64.

5. Aldea GS, Austin REJ, Flynn AE, Coggins DL, Husseini W, Hoffman JIE. Heterogeneous delivery of cardioplegic solution in the absence of coronary artery disease. J Thorac Cardiovasc Surg. 1990;99:345-53.

6. Bassingthwaighte J, King R, Roger S. Fractal nature of regional myocardial blood flow heterogeneity. Circ Res. 1989;65:578-90.

7. Bassingthwaighte J, Van Beek J, King R. Fractal branchings: the basis of myocardial flow heterogeneities? Ann N Y Acad Sci. 1990;591:392401.

8. Bassingthwaighte JB, Beard DA. Fractal $15_{\mathrm{O}}$-labeled water washout from the heart. Circ Res. 1995;77:1212-21.

9. Glenny RW, Robertson HT, Yamashiro S, Bassingthwaighte JB. Applications of fractal analysis to physiology. J Appl Physiol. 1991;70:2351-67.

10. Lefevre GR, Kowalski SE, Girling LG, Thiessen DB, Mutch WA. Improved arterial oxygenation after oleic acid lung injury in the pig using a computer-controlled mechanical ventilator. Am J Respir Crit Care Med. 1996;154:1567-72.

11. Mutch WA, Lefevre GR, Thiessen DB, Girling LG, Warrian RK. Computer-controlled cardiopulmonary bypass increases jugular venous oxygen saturation during rewarming. Ann Thorac Surg. 1998; 65:59-65.

12. Mutch WA, Eschun GM, Kowalski SE, Graham MR, Girling LG, Lefevre GR. Biologically variable ventilation prevents deterioration of gas exchange during prolonged anaesthesia. Br J Anaesth. 2000; 84:197-203.

13. Mutch WA, Harms S, Graham MR, Kowalski SE, Girling LG, Lefevre GR. Biologically variable or naturally noisy mechanical ventilation recruits atelectatic lung. Am J Respir Crit Care Med. 2000;162:319-23.

14. Mutch WAC, Warrian RK, Eschun GM, Girling LG, Doiron L, Cheang MS, et al. Biologically variable pulsation improves jugular venous oxygen saturation during rewarming. Ann Thorac Surg. 2000; 69:491-7.

15. Mutch WA, Harms S, Lefevre GR, Graham MR, Girling LG, Kowalski SE. Biologically variable ventilation increases arterial oxygenation over that seen with positive end-expiratory pressure alone in a porcine model of acute respiratory distress syndrome. Crit Care Med. 2000;28:2457-64.

16. Suki B, Alencar AM, Sujeer MK, Lutchen KR, Collins JJ, Andrade JS Jr, et al. Life-support system benefits from noise. Nature. 1998;393: 127-8.

17. Bernard F, Denault A, Babin D, Goyer C, Couture P, Couturier A, et al. Diastolic dysfunction is predictive of difficult weaning from cardiopulmonary bypass. Anesth Analg. 2001;92:291-8.

18. McKenney PA, Apstein CS, Mendes LA, Connelly GP, Aldea GS, Shemin RJ,et al. Increased left ventricular diastolic chamber stiffness immediately after coronary artery bypass surgery. J Am Coll Cardiol. 1994;24:1189-94.

19. Schreuder JJ, Biervliet JD, van der Velde ET, ten Have K, van Dijk AD, Meyne NG, et al. Systolic and diastolic pressure volume relationships during cardiac surgery. J Cardiothorac Vasc Anesth. 1991;5: 539-45.

20. Casthely PA, Shah C, Mekhjian H, Swistel D, Yoganathan T, Komer $\mathrm{C}$, et al. Left ventricular diastolic function after coronary artery bypass grafting: a correlative study with three different myocardial protection techniques. J Thorac Cardiovasc Surg. 1997;114:254-60.

21. Buckberg GD. Update on current techniques of myocardial protection. Ann Thorac Surg. 1995;60:805-14.

22. Chen YF, Wu SC, Huang CH, Pan PC, Lee CS, Lin CC. Morphometric identification of luminal narrowing of myocardial capillaries after cardioplegic arrest. Ann Thorac Surg. 2001;71:243-8.

23. Austin REJ, Aldea GS, Coggins DL, Flynn AE, Hoffman JI. Profound spatial heterogeneity of coronary reserve: discordance between patterns of resting and maximal myocardial blood flow. Circ Res. 1990;67:319-31.

24. Ghaleh B, Shen YT, Vatner SF. Spatial heterogeneity of myocardial blood flow presages salvage versus necrosis with coronary artery reperfusion in conscious baboons. Circulation. 1996;94:2210-5.

25. Mori F, Ivey TD, Itoh T, Thomas R, Breazeale DG, Misbach G. Effects of pulsatile reperfusion on postischemic recovery of myocardial function after global hypothermic cardiac arrest. J Thorac Cardiovasc Surg. 1987;93:719-27.

26. Chiang BY, Ye CH, Gou XD, Gu YJ, Zhou Y, Hong JP, et al. Effects of pulsatile reperfusion on globally ischemic myocardium. ASAIO J. 1993;39:M438-43.

27. Steed DL, Follette DM, Foglia R, Maloney JV, Buckberg GD. Effects of pulsatile assistance and nonpulsatile flow on subendocardial perfusion during cardiopulmonary bypass. Ann Thorac Surg. 1978;26:13341.

28. Levine FH, Phillips HR, Carter JE, Philbin DM, Osbakken MD, Boucher CA, et al. The effect of pulsatile perfusion on preservation of left ventricular function after aortocoronary bypass grafting. Circulation. 1981;64(Suppl):II-40-3 